\title{
Lymphocyte interphase chromatin in healthy subjects, patients with Down's syndrome, and their parents and sibs
}

\author{
K N FEDOROVA AND I E YUDINA \\ From the Institute of Medical Genetics, AMS USSR, Moscow.
}

SUMMARY A study of the interphase chromatin structure of lymphocytes in healthy subjects, patients with Down's syndrome, and their parents and sibs was carried out by AO labelled fluorometry using our modification of DNP cell thermal denaturation. Analysis by the Sperry Univac 90/30-B computer showed that in $40 \%$ of healthy subjects the lymphocyte chromatin melting profiles had a regularly repeated curve with six (seven) maxima at definite temperatures. In the remaining $60 \%$ some regularly repeated deviations were present and were correlated with the sex of the subject examined. There were five subgroups in the female group and seven subgroups in the male group.

In $97 \%$ of patients with Down's syndrome the lymphocyte chromatin melting profiles gave curves with three maxima at temperatures of 65,85 , and $92^{\circ} \mathrm{C}\left( \pm 2^{\circ}\right)$. Maxima at 78 and $45^{\circ} \mathrm{C}$ were absent.

In $80 \%$ of the mothers of probands with Down's syndrome and in $30 \%$ of female sibships, lymphocyte melting profiles also produced curves with three maxima: 65,85 , and $92^{\circ} \mathrm{C}\left( \pm 2^{\circ}\right)$. In view of the fact that similar changes were observed in mothers and female sibs only, we propose that some women may have genotypical peculiarities which may possibly contribute to the origin of this chromosome pathology.

The purpose of the investigation described here was to develop a new approach to research into the structure of the interphase chromatin in human cells. By this approach we were able to carry out a comparative analysis of the correlation between karyotype, phenotype, and genotype in healthy subjects, patients with trisomy 21 , and their parents and sibs.

\section{Materials and methods}

Using a comparative analysis of temperature dependent fluorescence of acridine orange bound to cellular DNP by our modification ${ }^{1}$ of the method of Ringertz and Gledhill, ${ }^{2}$ the structure of the interphase chromatin of peripheral blood lymphocytes was examined in the following groups of subjects.

(1) Healthy donors: 164 subjects of whom 98 were males and 66 were females, age range 20 to 45 years.

(2) Fifty patients with Down's syndrome $(2547, X Y$, +21 and $2547, X X,+21$ ), age range 8 to 30 years.

(3) Parents of children with Down's syndrome:
50 mothers aged 20 to 48 and 35 fathers aged 20 to 58.

(4) Sibs: 15 girls aged 6 to 22 and 15 boys aged 10 to 24 .

Changes of the chromatin structure in response to heating were followed up by the amount of fluorescent label, acridine orange (AO), taken up, with the intervals of reading at 1 to $2^{\circ} \mathrm{C}$.

Investigations were carried out using short term cell cultures, that is, cells incubated for one hour in a nutrient medium with $10 \%$ autologous serum.

The fluorescence intensity of AO bound to the lymphocyte chromatin DNA was measured by the Opton MSP-0.5 scanning microscope-photometer. Fluorescence was excited with light of wavelength $(\lambda)$ $365 \mathrm{~nm}$ and recorded at $\lambda=530 \mathrm{~nm}$ by means of interference light filter. Changes in intensity of fluorescence at $\lambda=640 \mathrm{~nm}$ were recorded simultaneously to define ' $\alpha$ ' ratio $=F_{640} / F_{530}$. The $F_{640}$ value was negligible. Therefore, only the results of changes for $F_{530}$ are given. The instrument operational details, as well as lymphocyte isolation and 
cell cultivation, were described by us earlier. ${ }^{1}$ The results of fluorescence intensity $\left(F_{530}\right)$ of the DNP $=$ AO complex of cells in a temperature range of 20 to $100^{\circ} \mathrm{C}$ were examined using the Sperry Univac 90-30-B" computer with a special programme. This was done in order to determine: (1) the statistical significance of the maxima demonstrated at certain temperature intervals; and (2) to distribute into groups all the numerical values of the curves obtained in relation to the coinciding characteristics of the presence or absence of maxima.

Numerical values of the $F_{530} \quad D N P=A O$ complex in 24 points corresponding to the following temperatures: $20,35,40,42,45,47,50,55,60,65$, $67,70,75,77,78,80,82,85,87,88,89,90,92$, and $95^{\circ} \mathrm{C}$ were used as initial data for the programme. Each curve was examined with respect to the presence of a maximum in the environs of points corresponding to $45,65,78,88$, and $92^{\circ} \mathrm{C}$. These environs were: $42,47,60,67,77,78,85,90$, and $92^{\circ} \mathrm{C}$. On a fixed curve the characteristics of the presence of a maximum were determined for each of these points: $\mathrm{M}$-maximum, $\mathrm{N}$-absence of maximum, $\mathrm{V}$ uncertainty (absence of data). For this purpose the presence of a maximum was first analysed in each point of the environ separately, the value of a curve being examined in the point analysed and in two adjacent ones. If the value in this point exceeded those in the adjacent ones by more than 2 , the presence of a (mild: $\pm 2^{\circ}$ ) maximum was noted. In all other cases no maximum was considered to be present. This procedure was applied to all points of the environ. Then all curves were broken up into classes or groups of curves with coinciding characteristics of the presence of maxima $M$ (or $N$ ) in six environs.

\section{Results}

\section{HEALTHY SUBJECTS}

A computerised analysis of the data obtained $\left(F_{530}\right.$ DNP-AO complex in the temperature range of 20 to $100^{\circ} \mathrm{C}$ ) showed that, irrespective of sex, in about $40 \%$ of cases $( \pm 0.35)$ the temperature dependent variations in AO fluorescence of interphase chromatin in healthy subjects presented a complicated but regularly repeated picture of a curve with six maxima at the following temperatures: $45,65( \pm 3)$, $78( \pm 1), 85,88$, and $92( \pm 2)^{\circ} \mathrm{C}$, that is, a clear-cut 'identity' of the above parameter was common to different subjects. The regularity of DNP cell melting profiles was revealed in the most numerous group of healthy subjects, $50 \%$ of females and $40 \%$ of males (modal class), and was taken by us as the standard normal variant (fig 1).

In the remaining $60 \%$ of cases, various but regularly repeated deviations were noted: (1) the absence of a maximum; (2) the merging of two adjacent maxima in one; and (3) the shift of one ofo the maxima to the left or to the right by more than $3^{\circ} \mathrm{C}$.

It should be noted that in most cases the nature of the deviations depended on the sex of the subject examined. In females the most common type of deviation was the merging of two maxima into one:

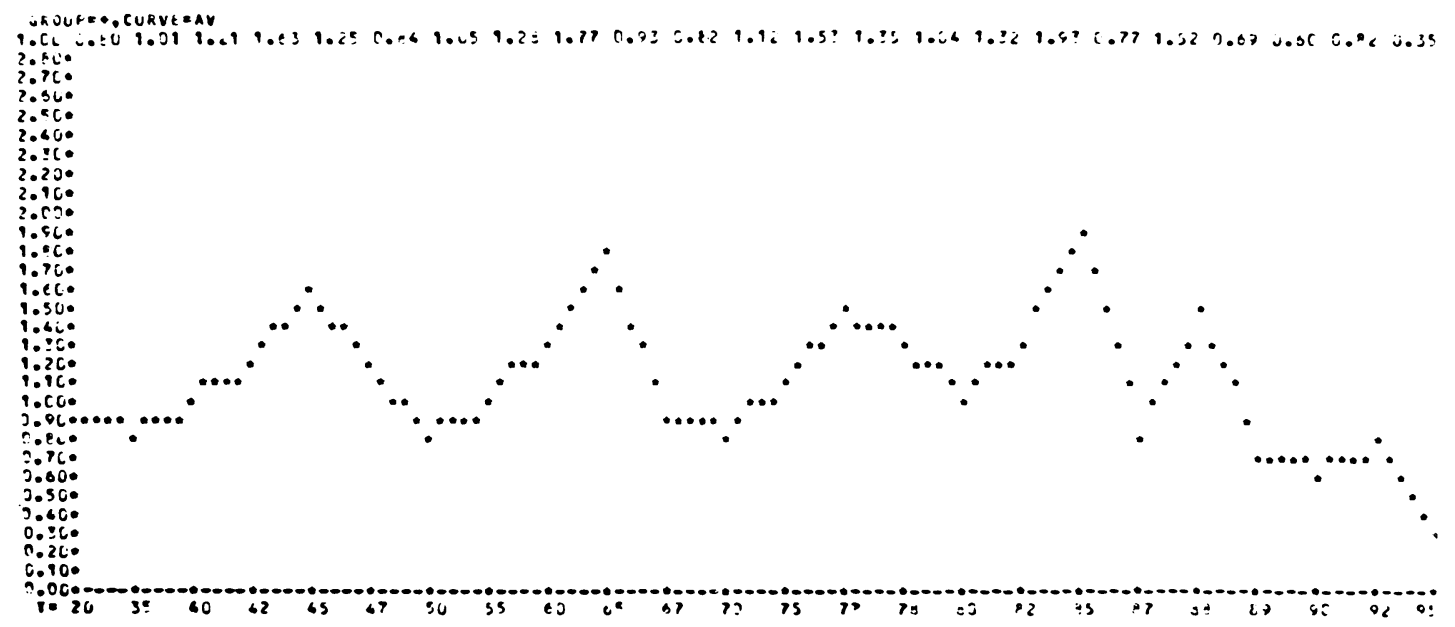

FIG 1 Lymphocyte interphase chromatin melting profiles of healthy subjects $(46 \%)$ obtained by acridine orange fuorescence $\left(F_{530}\right)$. 
78 and $85^{\circ} \mathrm{C}$ (nine cases), 85 and $88^{\circ} \mathrm{C}$ (eight cases), and 88 and $92^{\circ} \mathrm{C}$ (nine cases) (fig 2). As for the coinciding characteristics of the presence of the maxima on the curve of DNP lymphocyte melting, the female control group fell into five subgroups, each containing not less than five identical cases.

Seven subgroups were singled out in the control group of males: the basic group comprised cases with six (seven) maxima revealed at definite temperatures $(-40 \%)$. The most common type of deviation was the absence of a maximum at $85^{\circ} \mathrm{C}$ and its appearance at $82^{\circ} \mathrm{C}(25 \%$ of the cases) (fig 3). There were unclassified, strictly individual types of deviations in the female group $(5 \%)$ and in the male group $(17 \%)$.

We were thus able, by using a computer, to classify individual cases of interphase chromatin structure by heat denaturation patterns in healthy subjects.

PATIENTS WITH DOWN'S SYNDROME

In response to heat the fluorescence intensity of $\mathrm{AO}$ combined with aneuploid genome chromatin increased regularly by 1.5 to 2 times at the following

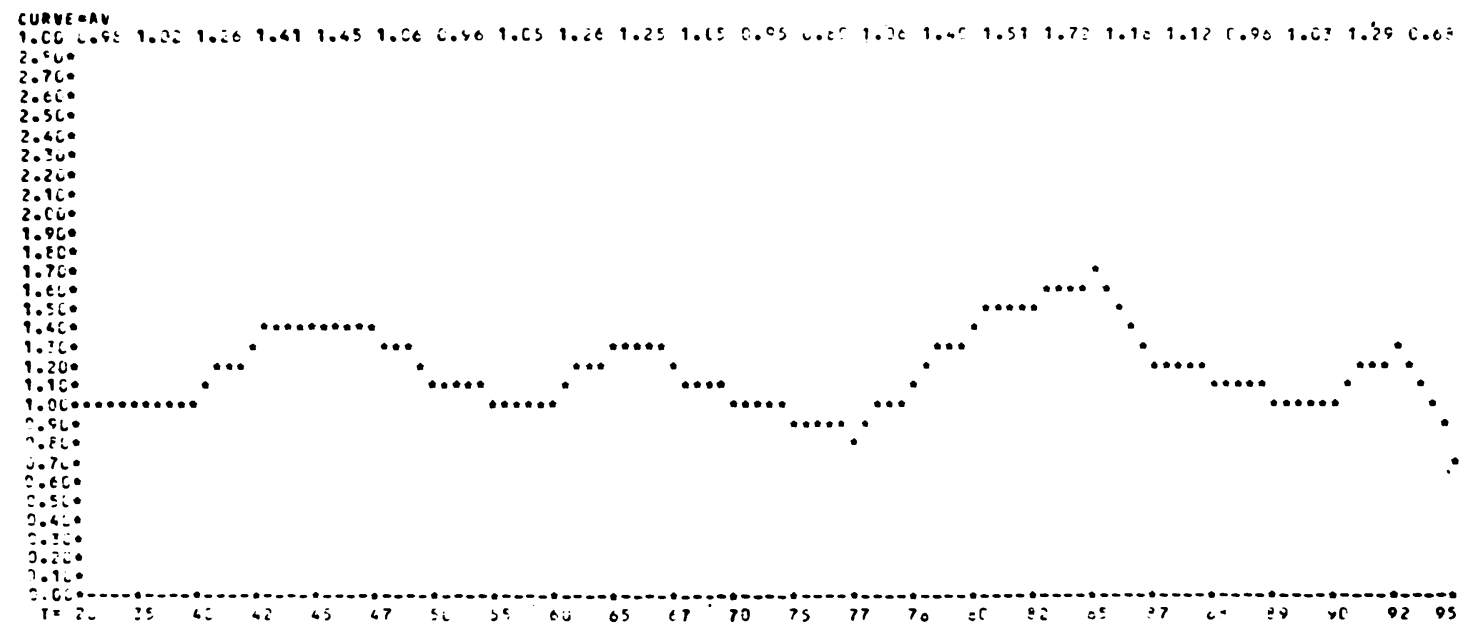

FIG 2 The most frequent modification of deviations in the lymphocyte interphase chromatin melting profiles of healthy females manifested in the merging of two maxima into one blurred maximum at 78 and $85^{\circ} \mathrm{C}(8 \%)$.

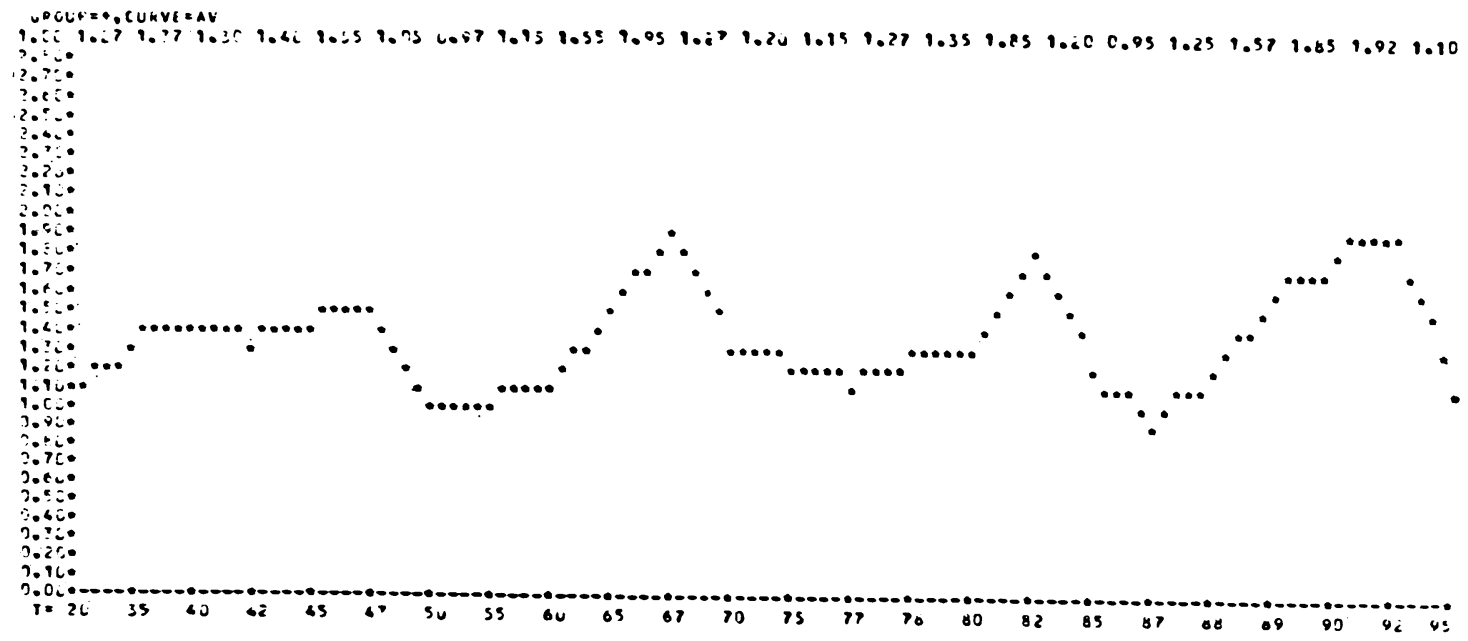

FIG 3 The most frequent modification of deviations in the lymphocyte interphase chromatin melting profiles of healthy males manifested in the absence of a maximum at $85^{\circ} \mathrm{C}(25 \%)$. 
temperatures: 65,85 , and $92^{\circ} \mathrm{C}\left( \pm 2^{\circ} \mathrm{C}\right)$, demonstrating only three maxima in contrast to the six or seven in the controls, regardless of the sex of the subject examined (fig 4).

It should be noted that the major differences in the curves of acridine orange fluorescence against temperature of diploid (healthy donors) and trisomic cells were within the temperature range of 70 to $85^{\circ} \mathrm{C}$. Within this temperature interval the patients' cells showed a smooth rise of the curve with a single blurred maximum in the region of $85^{\circ} \mathrm{C}$. In $95 \%$ of the cases there was no peak at $45^{\circ} \mathrm{C}$.

\section{MOTHERS OF PROBANDS WITH DOWN'S} SYNDROME AND FEMALE SIBS

In response to heat the lymphocytes of healthy mothers and female sibs $(80 \%$ and $30 \%$ of the cases respectively) reacted by a regular increase in the $\mathrm{AO}$ fluorescence intensity in the temperature ranges of $45,65,85$, and $92^{\circ} \mathrm{C}\left( \pm 2^{\circ} \mathrm{C}\right)$. Three or four maxima were demonstrable, as was typical of the probands. The maximum at $45^{\circ} \mathrm{C}$ was either absent or merged with the peak at $65^{\circ} \mathrm{C}$ (fig 5).

\section{FATHERS OF PROBANDS WITH DOWN'S}

SYNDROME AND MALE SIBS

No deviations from the standard were demonstrated in the fathers and their healthy sons (fig 6). Thus, certain signs of the changed interphase chromatin structure of patients with Down's syndrome were revealed only in their mothers and healthy female sibs. It is interesting to note that the main indication of the changed chromatin structure in patients with Down's syndrome (double maxima) was also present
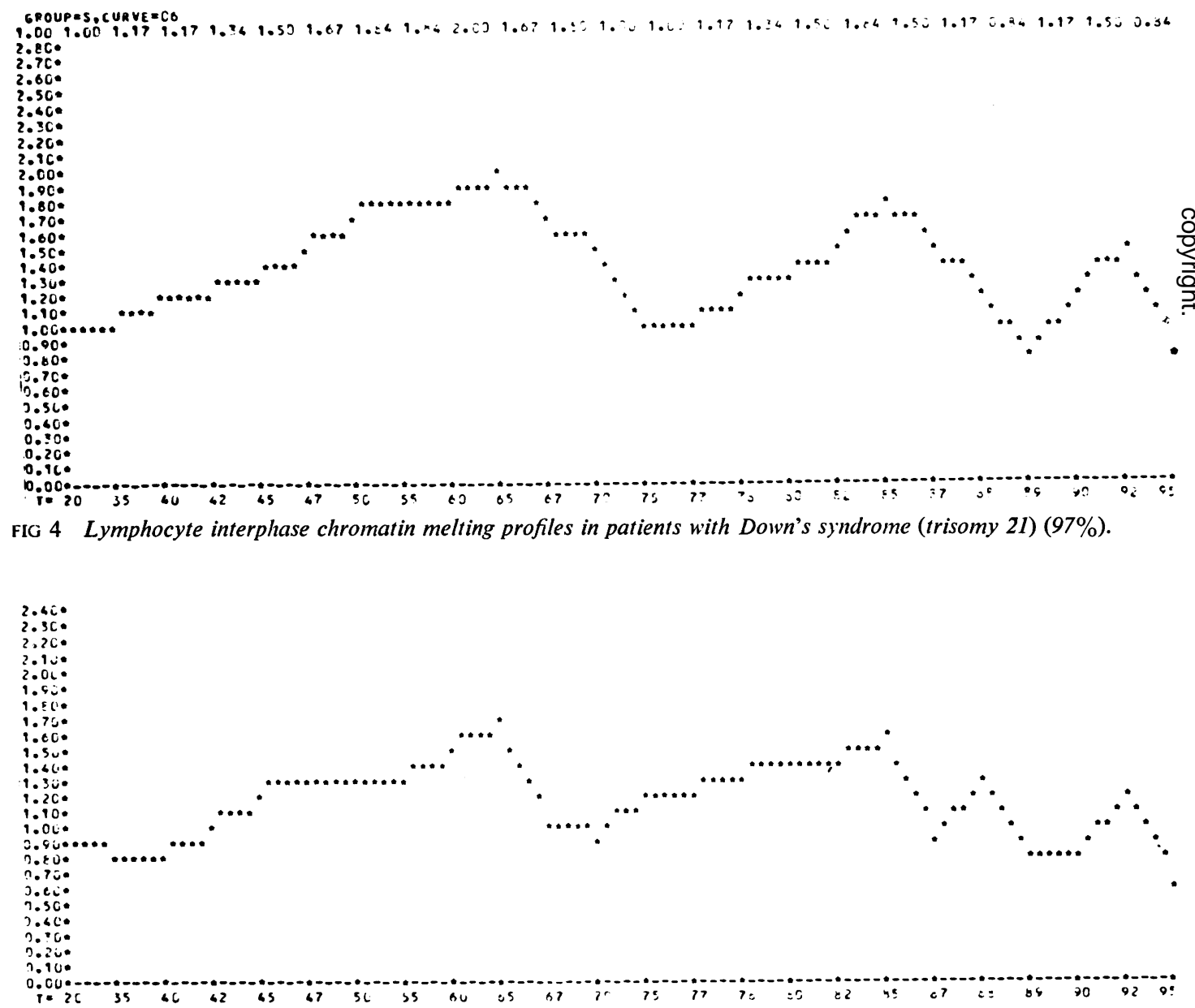

FIG 5 Lymphocyte interphase chromatin melting profiles of the healthy mother of a child with Down's syndrome. 

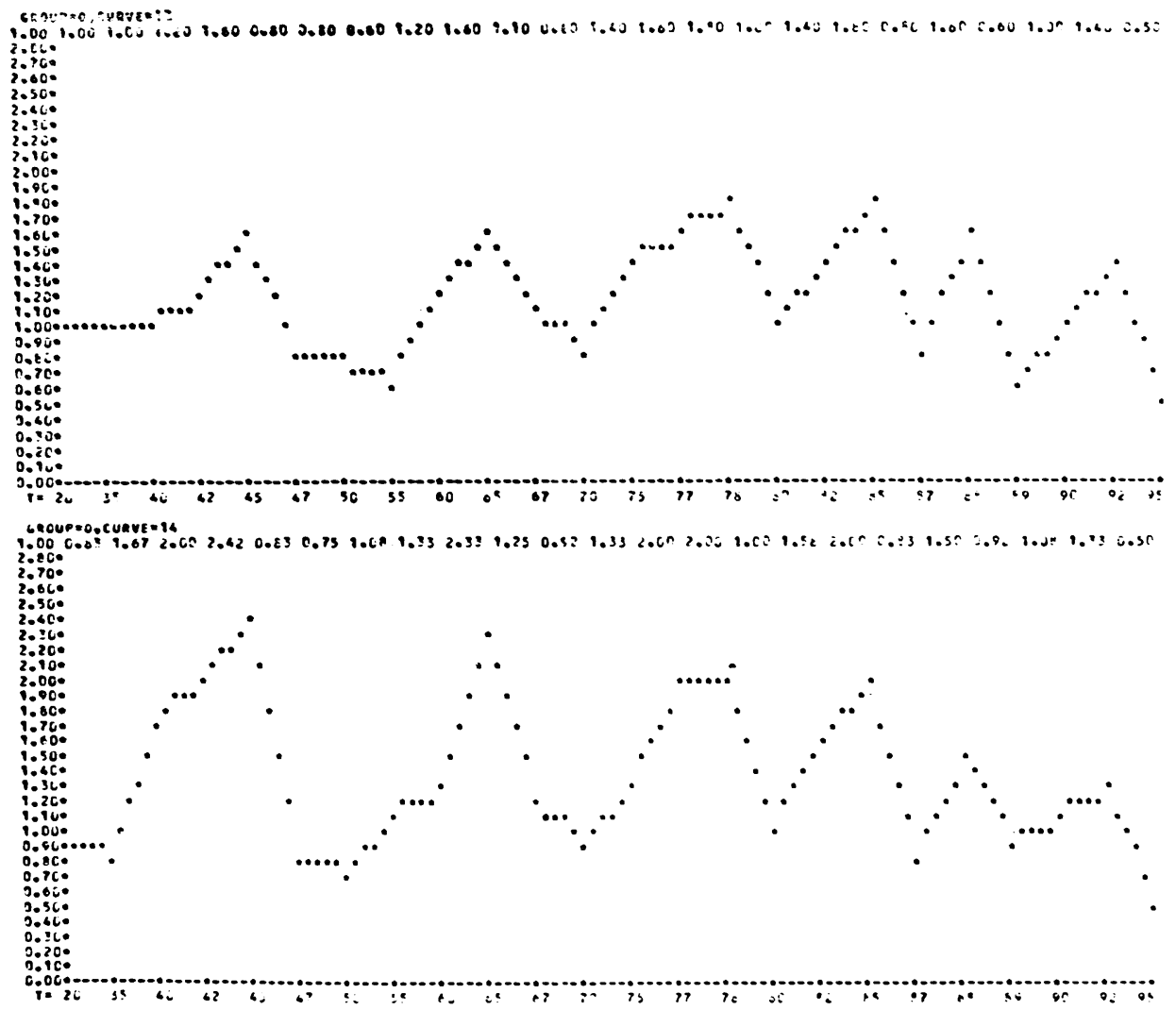

FIG 6 Lymphocyte interphase chromatin melting profiles of fathers (above) and male sibs (below). Abscissu, temperature (in ${ }^{\circ} \mathrm{C}$ ). Ordinate, ratio of the fluorescence intensity of $A O$, combined with human lymphocyte chromatin at a given temperature, to $A O$ fluorescence intensity at $20^{\circ} \mathrm{C}: \mathrm{F}_{530} \mathrm{~T}^{\circ} \mathrm{C} / \mathrm{F}_{530} 20^{\circ} \mathrm{C}$.

in a proportion ( $8 \%$ ) of the healthy female population. The above sign is not correlated with mother's age or with the sequence of birth (before or after the proband) in female sibs.

No change in chromatin structure, characteristic of patients with Down's syndrome, was demonstrated in the group of fathers and male sibs. Since this phenomenon did not show any correlation between probands, their fathers, and male sibs in the analysis of family cases, we suggest that such a change in the hereditary substrate structure may be a characteristic of the female genotype only.

\section{Discussion}

A study has been made of the interphase chromatin structure of lymphocytes in healthy subjects, patients with Down's syndrome, and their parents and sibs by studying thermally induced changes in acridine orange fluorescence of chromatin. The results show that in $40 \%$ of healthy normal subjects, irrespective of the sex of the subject examined, a clear picture is obtained. The changes in lymphocyte chromatin fluorescence with temperature show a regularly repeated pattern made up of a curve with six or seven maxima at different temperatures. In the remaining $60 \%$, various regularly repeated deviations are observed and are correlated with the sex of the subject. There are five subgroups among females and seven subgroups among males.

For patients with Down's syndrome, however, $97 \%$ of the lymphocyte chromatin fluorescence profiles give curves with maxima at only three temperatures: 65,85 , and $92^{\circ} \mathrm{C}\left( \pm 2^{\circ} \mathrm{C}\right)$. Maxima at $78^{\circ} \mathrm{C}$ and $45^{\circ} \mathrm{C}$ are absent. Furthermore, $80 \%$ of the mothers of probands with Down's syndrome and $30 \%$ of their female sibs also show maxima at these three temperatures. Male sibs and the fathers of Down's syndrome probands, however, show fluorescence profiles which do not differ from those of normal subjects.

We believe that, since the changed chromatin 
structural organisation we have observed in our experiments is only revealed in the mothers and some female sibs of Down's syndrome patients, there may exist a certain group of subjects within the female population whose genotypical pecularities are related to the aetiology of trisomy 21 .

The nature of the thermally induced chromatin changes revealed by acridine orange (AO) in our studies are, however, not entirely understood. The ratio of red to green acridine orange fluorescence has been widely used as a measure of DNA denaturation to melting, ${ }^{3}$ but in the present study this ratio remained constant and only the total fluorescence varied. The phenomenon observed does not, therefore, appear to represent actual denaturation of DNA. Displacement of proteins from DNA at higher temperatures, thereby unmasking dyebinding groups, could lead to increased AO binding and fluorescence, but this does not explain the decreases in fluorescence which were also observed. The true explanation of the phenomena observed must therefore await the results of further work. Meanwhile, it must be noted that, whatever the explanation, the thermally induced chromatin changes revealed by AO fluorescence are consistent within the different classes of subjects studied and are believed to represent some characteristic of the chromatin organisation in these classes.

If there is a group of high risk women who can be ascertained simply by AO fluorescence of their lymphocyte chromatin, then this technique could make an important contribution to the counselling of the female population in respect of their likelihood of producing a Down's syndrome child.

\section{References}

1 Fedorova $\mathrm{KN}$. The structure of the human cell interphase chromatin in the mosaic form of Down's syndrome. $J$ Ment Defic Res 1977;21:85-93.

2 Ringertz N, Gledhill B, Changes in DNP during spermatogenesis in the cell sensitivity of DNA to heat denaturation. Exp Cell Res 1970;62:204-18.

${ }^{3}$ Rigler R. Microfluorometric characterization of intracellular nucleic acids and nucleoproteins by acridine orange. Acta Physiol Scand 1966;67 :suppl 267.

Requests for reprints to Dr K Fedorova, Institute of Medical Genetics, AMS USSR, Kashirskoye Shosse 6A, 115478 Moscow, USSR. 